\title{
Performance of Selection Relaying and Cooperative Diversity
}

\author{
Abdulkareem Adinoyi, Member, IEEE, Yijia Fan, Member, IEEE, Halim Yanikomeroglu, Member, IEEE, \\ H. Vincent Poor, Fellow, IEEE, and Furaih Al-Shaalan
}

\begin{abstract}
In this paper, a two-hop cooperative multi-relay communication network is considered. Selection relaying schemes are attracting considerable attention due to their prudent bandwidth utilization and ability to provide full diversity. The recent developments in selection relaying (SR) have largely focused on information theoretic analyses such as outage performance. Some of these analyses are accurate only in high SNR regimes. This paper provides exact outage and capacity performance expressions for selection relaying and tight approximation over a sufficiently wide range of $S N R$ regimes for selection cooperative relaying. The $\epsilon$-outage capacity for $S R$ is also provided. The motivation for this work is that practical systems operate at far lower SNR values than those supported by the high SNR analysis. Therefore, designers should be able to evaluate system performance to a reasonable degree of accuracy over practical SNR values. Simulations are used to corroborate the analytical results and close agreement is observed.
\end{abstract}

Index Terms-Cooperative diversity, relay selection, outage probability and capacity.

\section{INTRODUCTION AND MOTIVATION}

I NTEREST in various forms of cooperative schemes has been growing steadily since the seminal work in cooperation diversity in [1]. This work re-examines the manner in which nodes relate to each other to provide a cost-effective way of harnessing the diversity advantages of multi-antenna systems without necessarily putting these antennas in one location. The literature has described this paradigm shift as cooperative relaying or user cooperation diversity, and it is particularly attractive for small-size, antenna-limited wireless devices. Infrastructure (fixed) relays are particularly appealing to wireless network service providers due to the challenges facing terminal cooperation.

Parallel relay cooperation can provide full diversity order by adopting protocols such as selective repetition relaying. In a repetition protocol-based two-hop multi-relay scheme, all relays retransmit the same (source) signal in round robin fashion. This is not bandwidth efficient since relays require

Manuscript received January 30, 2009; revised June 17, 2009; accepted August 14, 2009. The associate editor coordinating the review of this letter and approving it for publication was H. Dai.

A. Adinoyi is with Swedtel Arabia as a consultant for Saudi Telecom Company (STC), Riyadh, Saudi Arabia (e-mail: adinoyi@ieee.org).

H. Yanikomeroglu is with the Department of Systems and Computer Engineering, Carleton University, Ottawa, ON, Canada (e-mail: halim@sce.carleton.ca).

Y. Fan and H. V. Poor are with the Department of Electrical Engineering, Princeton University, Princeton, NJ, USA (e-mail: \{yijiafan, poor\}@ princeton.edu).

F. Al-Shaalan is with the Department of Electrical Engineering, King Saud University and STC, Riyadh, Saudi Arabia (e-mail: falshalan@yahoo.com).

Partial support was provided by the U.S. National Science Foundation under Grant CNS-06-25637. This work was presented in part at the IEEE Vehicular Technology Conference (VTC'08-Fall), Calgary, Alberta, September 2008.

Digital Object Identifier 10.1109/TWC.2009.12.090138 separate channels in the form of orthogonal time or frequency slots. The bandwidth inefficiency of such schemes could make these schemes unattractive, especially for a large number of relays. Selection relaying, on the other hand, provides full diversity without the excessive demand on bandwidth, provided the relay selection is performed carefully [2]-[5].

The present paper considers selection relaying that involves upper layers of the communication protocol in selecting a relay among multiple relays to assist the source. In other words, the selection diversity is an inherent feature in the routing opportunities offered by the relays. We provide performance analyses that are sufficiently accurate in both low and high SNR regions for such multi-relay networks. The outage probability and capacity expressions are exact for selection relaying and tight approximations for selection cooperative relaying. An important earlier work [3] has not considered outage and ergodic capacities, and bit error rate (BER). Another recent and relevant contribution to the topic of this paper can be found in [6]. The motivation for accurate performance estimation at low or medium SNR levels is the following. It is noted that high SNR analysis has theoretical merits; however, practical systems often operate at lower SNR values. Thus, it is desirable to be able to evaluate system performance to a reasonable degree of accuracy in the low SNR region for the purpose of network design and deployment.

\section{System AND Channel Models}

The system investigated in this paper is shown in Fig. 1. The nodes - source, destination, and relays - are denoted as $\mathrm{S}, \mathrm{D}$, and $\mathrm{R}$, respectively. There are $N$ fixed single-antenna relays in the network. In the half-duplex two-hop protocol, the best relay assists S-D communication. The treatment in this paper assumes that the destination uses the signal received through the relay according to the following methods: selection relaying (SR), where the S-D path is not usable at the destination and selection cooperative relaying (SCR), where the S-D link is used at the destination.

Both slow and fast fading channels are considered. Independent and identically distributed Rayleigh fading is assumed across all links. The received signal at node $j$ transmitted from node $i$ is written as $y_{i j}=\alpha_{i j} \chi_{i}+n_{j}$, where $\chi_{i}$ is the signal transmitted from node $i, \alpha_{i j}$ is the channel gain between $i$ and $j$, and $n_{j}$ is additive white Gaussian noise (AWGN) at $j$. The instantaneous SNR for the link $i$ and $j$ is $\gamma_{i j}=\alpha_{i j}^{2} E_{s} / N_{0}$, where $E_{s}$ is the transmit energy per symbol, and $N_{0}$ is the AWGN power spectral density. Therefore, the average SNR is given by $\bar{\gamma}_{i j}=E\left[\gamma_{i j}\right]$.

The first hop transmission can be characterized by the 


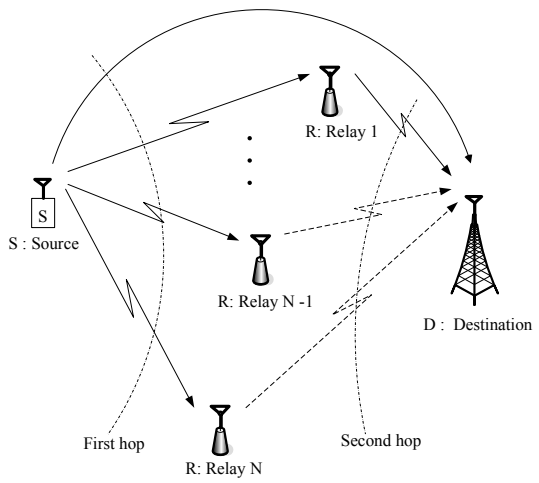

Fig. 1. A multi-relay network with best relay selection.

following relation:

$$
y_{\mathrm{s} j}=\alpha_{\mathrm{s} j} \chi_{\mathrm{s}}+n_{j}, \quad j=1,2, \cdots, N .
$$

If the destination can hear the source, the direct signal can be expressed as

$$
y_{\mathrm{SD}}=\alpha_{\mathrm{SD}} \chi_{\mathrm{s}}+n_{\mathrm{D}} .
$$

The best relay forwards to the destination in the second hop, which can be expressed as

$$
y_{j^{*} \mathrm{D}}=\alpha_{j^{*} \mathrm{D}} \tilde{\chi}_{\mathrm{s}}+n,
$$

where $\tilde{\chi}_{\mathrm{s}}$ is the regenerated signal at the relay and $j^{*}$ stands for the selected best relay.

The relay selection, which is essentially a routing problem, is based on backward and forward channels of relays, and this is performed jointly as follows:

- For each relay link, select across the hops:

$$
\Omega_{j}=\min \left\{\gamma_{s j}, \gamma_{j D}\right\} \text { for } j=1,2, \cdots, N,
$$

- Select across the relays:

$$
\gamma_{j^{*}}=\max _{j=1,2, \ldots, N}\left\{\Omega_{j}\right\} .
$$

We refer to the minimum SNR of the best relay as $\gamma_{1}$, i.e., $\gamma_{1}=\gamma_{j^{*}}$. Similarly, we denote the instantaneous SNR of the S-D link as $\gamma_{0}$. For Rayleigh fading channels, $\gamma_{0}$ is an exponential random variable, and its expected value is denoted by $\overline{\gamma_{0}}$. The statistics of $\gamma_{1}$ are discussed below.

It should be mentioned that in fast fading channels, relay selection will incur higher complexity compared to slowly varying channels due to frequent switching and signaling to facilitate the selection.

\section{Performance Analysis}

The notion of capacity is valid in ergodic (fast fading) channels where no constraints are imposed on the decoding delay at the receiver. However, in slow fading channels, outage capacity or outage probability is the relevant performance measure. In this section we provide analytical expressions for calculating the ergodic capacity (valid for fast fading channels) and outage probability (valid for slow fading channels) for SCR and SR schemes. In addition, the outage capacity is given for SR. While these performance measures are exact for SR they are tight approximations for SCR. Finally, approximate BER expressions are provided for the two schemes.

\section{A. Selection Relaying (SR)}

The selection of the best relay requires knowledge of order statistics. The first step is to obtain the weaker link between the first hop and second hop of each relay node. These weak links are ordered and the one with the highest SNR is selected as the candidate to relay the message to the destination as explained above. Given a probability density function $(\mathrm{PDF})^{1}, f(\gamma)$, and cumulative density function (CDF), $F(\gamma)$, for the individual channel gains the PDF of such ordered random variables can be derived [8] [9] and is given as $\left.p(\gamma)=2 N f(\gamma)[1-F(\gamma))]\left[2 F(\gamma)-(F(\gamma))^{2}\right)\right]^{N-1}$. For the underlying Rayleigh distributed random variable with $f(\gamma)=\frac{1}{\bar{\gamma}} \exp \left(\frac{-\gamma}{\bar{\gamma}}\right)$, and $F(\gamma)=1-\exp \left(\frac{-\gamma}{\bar{\gamma}}\right)$, the PDF of $\gamma_{1}$ can be obtained as

$$
\begin{aligned}
p\left(\gamma_{1}\right) & =N \frac{\exp \left(-\frac{\gamma_{1}}{\overline{\gamma_{1}} / 2}\right)}{\overline{\gamma_{1}} / 2}\left(1-\exp \left(-\frac{\gamma_{1}}{\overline{\gamma_{1} / 2}}\right)\right)^{N-1} \\
& =\sum_{i=1}^{N}(-1)^{i-1}\left(\begin{array}{c}
N \\
i
\end{array}\right) \frac{2 i}{\overline{\gamma_{1}}} \exp \left(-i \frac{2 \gamma_{1}}{\overline{\gamma_{1}}}\right) .
\end{aligned}
$$

The second part of (4) is obtained through binomial expansion.

1) Outage probability for $S R$ : An outage is defined as the event in which the communication channel does not support a target data rate. Using the PDF in (4) the exact outage performance for SR can be evaluated as follows (see Appendix I):

$p_{\text {out, SR }}=\sum_{i=1}^{N}(-1)^{i-1}\left(\begin{array}{c}N \\ i\end{array}\right)\left(1-\exp \left[-\frac{2 i\left(2^{2 \mathcal{R}}-1\right)}{\bar{\gamma}}\right]\right)$,

where $\mathcal{R}$ is the target rate. An alternative performance measure to characterize slow fading channels is the $\epsilon$-outage capacity $\left(C_{\epsilon}\right)$. This is the largest (realizable) transmission rate such that the outage is less than a certain $\epsilon$. This metric is derived in Appendix III and given as

$$
C_{\epsilon}=\frac{1}{2} \log _{2}\left(1+\frac{\bar{\gamma}}{2} \log \left(\frac{1}{1-\epsilon^{\frac{1}{N}}}\right)\right) \quad \text { bits } / \mathrm{s} / \mathrm{Hz} .
$$

2) Ergodic capacity for SR: This quantity can be shown (see Appendix II) to be given by

$$
\tilde{C}=\sum_{i=1}^{N}(-1)^{i-1}\left(\begin{array}{c}
N \\
i
\end{array}\right) \frac{1}{\ln 2} \exp \left(\frac{2 i}{\bar{\gamma}}\right) E_{1}\left(\frac{2 i}{\bar{\gamma}}\right) \text { bits/s/Hz, }
$$

where $E_{1}[\cdot]$ is the exponential integral [10].

3) Probability of bit error calculation for SR: In SR relaying the direct path is unusable due to deep fade instances or heavy shadowing. Hence, the BER performance can be derived from the expression given in Appendix III by letting $\gamma_{0} \rightarrow-\infty$ in (15). After some manipulation, the BER for binary phase shift keying (BPSK) signaling can be obtained as

$$
B E R_{\mathrm{SR}} \approx \frac{1}{4} \sum_{i=1}^{N}(-1)^{i-1}\left(\begin{array}{c}
N \\
i
\end{array}\right) B_{z_{1}}\left[1, \frac{1}{2}\right],
$$

where $B_{a}[\cdot, \cdot]$ is the incomplete beta function [10]. It can be observed that (8) is a strikingly simple and compact expression.

\footnotetext{
${ }^{1} f$ denotes the PDF of $\gamma_{i j}$ and $p$ denotes that of $\gamma_{1}=\gamma_{j^{*}}$.
} 


\section{B. Selection Cooperation Relaying (SCR)}

SCR assumes that the best relay is not in outage. Therefore, the maximal ratio combining (MRC) receiver combines the direct and the relay link SNRs. The relay link SNR is approximated as the weaker of the first and second hop instantaneous SNR of the selected relay. The relay cooperation is effective only when the achieved rate on the source-best relay link is greater than that on the source-destination link [11], a condition that can easily be satisfied in an asymmetric relay network, where relays can be placed strategically to maintain a strong link to the source. Since MRC is adopted, the combined SNR can be written as $\beta=\gamma_{0}+\gamma_{1}$. Due to the independence of $\gamma_{0}$ and $\gamma_{1}$, the PDF of $\beta$ can be obtained through the convolution of the PDFs of $\gamma_{1}$ and $\gamma_{0}$, i.e.,

$$
p(\beta)=\int_{0}^{\beta} p_{\gamma_{0}}(\tau) p_{\gamma_{1}}(\beta-\tau) d \tau .
$$

Using (4) and the PDF of $\gamma_{0}$ (i.e., $\frac{1}{\bar{\gamma}_{0}} \exp \left(-\frac{\gamma_{0}}{\bar{\gamma}_{0}}\right)$ ), we have

$$
\begin{aligned}
p(\beta) & =\int_{0}^{\beta} \sum_{i=1}^{N}(-1)^{i-1}\left(\begin{array}{c}
N \\
i
\end{array}\right) \frac{2 i}{\overline{\gamma_{1}} \overline{\gamma_{0}}} \\
& \times \exp \left(-\frac{i \tau}{\overline{\gamma_{1}} / 2}\right) \exp \left(-\frac{\beta-\tau}{\bar{\gamma}_{0}}\right) d \tau .
\end{aligned}
$$

By interchanging the integral and summation, (10) can be expressed as

$$
\begin{aligned}
p(\beta) & =\sum_{i=1}^{N}(-1)^{i-1}\left(\begin{array}{c}
N \\
i
\end{array}\right) \frac{2 i}{\overline{\gamma_{1}} \overline{\gamma_{0}}} \\
& \times \exp \left(-\frac{\beta}{\bar{\gamma}_{0}}\right) \int_{0}^{\beta} \exp \left(-\left[\frac{i \tau}{\overline{\gamma_{1}} / 2}-\frac{\tau}{\bar{\gamma}_{0}}\right]\right) d \tau .
\end{aligned}
$$

Finally, evaluating the integral and tidying up, (11) simplifies to

$$
\begin{aligned}
p(\beta) & =\sum_{i=1}^{N}(-1)^{i-1}\left(\begin{array}{c}
N \\
i
\end{array}\right) \frac{2 i}{2 i \overline{\gamma_{0}}-\overline{\gamma_{1}}} \\
& \times\left(\exp \left[-\frac{\beta}{\bar{\gamma}_{0}}\right]-\exp \left[-\frac{2 i \beta}{\overline{\gamma_{1}}}\right]\right) .
\end{aligned}
$$

The PDF obtained in (12) characterizes the SCR scheme and thus can be employed for deriving the expression for outage, average capacity, and probability of error. Again, these are approximate measures as the decoding error at the best relay is ignored.

1) Outage probability for SCR: The outage probability is bounded by the following expression (see Appendix I for the derivation):

$$
\begin{aligned}
p_{\text {out }, \text { SCR }} & \geq \sum_{i=1}^{N}(-1)^{i-1}\left(\begin{array}{c}
N \\
i
\end{array}\right)\left(1+\frac{1}{2 i \overline{\gamma_{0}}-\overline{\gamma_{1}}}\right. \\
& \left.\times\left[\overline{\gamma_{1}} \exp \left(-\frac{2 i a}{\overline{\gamma_{1}}}\right)-2 i \overline{\gamma_{0}} \exp \left(-\frac{a}{\bar{\gamma}_{0}}\right)\right]\right),
\end{aligned}
$$

where $\mathcal{R}$ is the target rate, and $a=2^{2 \mathcal{R}}-1$.
2) Ergodic capacity for the SCR: Since ergodic channels are assumed, averaging the instantaneous channel capacity over the fading distribution has operational meaning. The ergodic capacity of the SCR scheme, in bits/s/Hz, can be bounded as

$$
\begin{aligned}
\tilde{C} & \leq \int_{0}^{\infty} \frac{1}{2} \log _{2}(1+\beta) p(\beta) d \beta \\
& =\sum_{i=1}^{N}(-1)^{i-1}\left(\begin{array}{c}
N \\
i
\end{array}\right) \\
& \times\left(\frac{2}{\left(2 \overline{\gamma_{1}}-4 i \overline{\gamma_{0}}\right) \ln 2}\right)\left[2 i \overline{\gamma_{0}} \exp \left(\frac{1}{\overline{\gamma_{0}}}\right)\left(E_{1}\left[\frac{-1}{\overline{\gamma_{0}}}\right]\right)\right. \\
& \left.-\overline{\gamma_{1}} \exp \left(\frac{2 i}{\overline{\gamma_{1}}}\right)\left(E_{1}\left[\frac{-2 i}{\overline{\gamma_{1}}}\right]\right)\right] \\
& =\sum_{i=1}^{N}\left(\begin{array}{c}
N \\
i
\end{array}\right) \frac{(-1)^{i-1}}{\left(\overline{\gamma_{1}}-2 i \overline{\gamma_{0}}\right) \ln 2} \\
& \times\left[-2 i \overline{\gamma_{0}} \exp \left(\frac{1}{\overline{\gamma_{0}}}\right) E_{1}\left[\frac{1}{\overline{\gamma_{0}}}\right]+\overline{\gamma_{1}} \exp \left(\frac{2 i}{\overline{\gamma_{1}}}\right) E_{1}\left[\frac{2 i}{\overline{\gamma_{1}}}\right]\right] .
\end{aligned}
$$

3) BER calculation for SCR: The BER of BPSK is given (that of other modulation techniques can similarly be derived) using the PDF given above as

$$
\begin{aligned}
B E R_{\mathrm{SCR}} & \approx \frac{1}{2} \int_{0}^{\infty} \operatorname{erfc}(\sqrt{\beta}) p(\beta) d \beta \\
& =\frac{1}{2} \int_{0}^{\infty} \operatorname{erfc}(\sqrt{\beta}) \sum_{i=1}^{N}(-1)^{i-1}\left(\begin{array}{c}
N \\
i
\end{array}\right) \\
& \times \frac{2 i}{2 i \overline{\gamma_{0}}-\overline{\gamma_{1}}}\left(\exp \left[-\frac{\beta}{\bar{\gamma}_{0}}\right]-\exp \left[-\frac{2 i \beta}{\overline{\gamma_{1}}}\right]\right) d \beta \\
& =\sum_{i=1}^{N}(-1)^{i-1}\left(\begin{array}{c}
N \\
i
\end{array}\right) \\
& \times \frac{i}{2\left(2 i \overline{\gamma_{0}}-\overline{\gamma_{1}}\right)}\left[\overline{\gamma_{0}} B_{z_{0}}\left[1, \frac{1}{2}\right]-\frac{\overline{\gamma_{1}}}{2 i} B_{z_{1}}\left[1, \frac{1}{2}\right]\right],
\end{aligned}
$$

where $z_{0}=\frac{1}{\overline{\gamma_{0}+1}}, z_{1}=\frac{2 i}{\overline{\gamma_{1}+2 i}}$, and again $B_{x}[a, b]$ is the incomplete beta function.

\section{NUMERICAL EXAMPLES}

To explore the derived expressions numerically, we consider a semi-asymmetric network, in which all relay links have the same average SNR, which is different from that of the S-D link. The scenario in which the S-D path strength is weaker than those of the R-D or S-R paths represents an interesting situation because it exposes the path-loss reduction benefit of relays. In fact, this path-loss reduction benefit can be viewed as the primary objective of the use of relays. That is, in this scenario, relays act as intermediate nodes between the source and destination. The model and linear relay placement used in [13] and a path-loss exponent $\kappa=3$ are adopted.

The outage performance for the SCR and SR schemes is plotted for a semi-asymmetric network in which the relays are mid-way between the source and destination. It can be observed (by calculating the slope of the curves in Fig. 2) that full diversity is obtained. Figure 3 shows the outage performance of the SCR scheme for the balanced network (the S-D, S-R, and R-D links all have the same average 


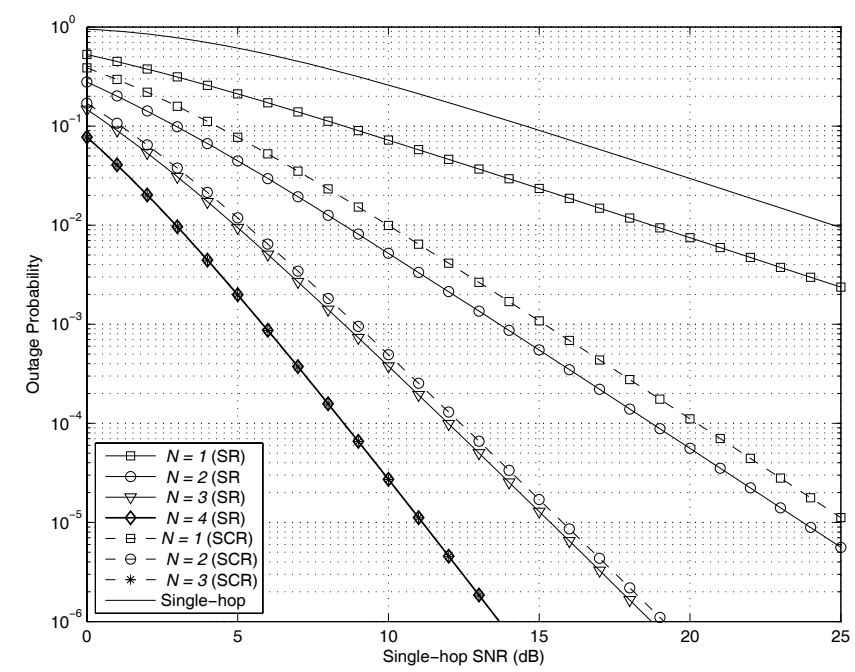

Fig. 2. Outage performance of the selection relaying (SR) and selection cooperative relaying (SCR) schemes in Rayleigh fading; the solid curves and asterisks represent SCR for $N=1,2$, and 3 relays, $\bar{\gamma}_{s r}=\bar{\gamma}_{r d}=8 \bar{\gamma}_{s d}$.

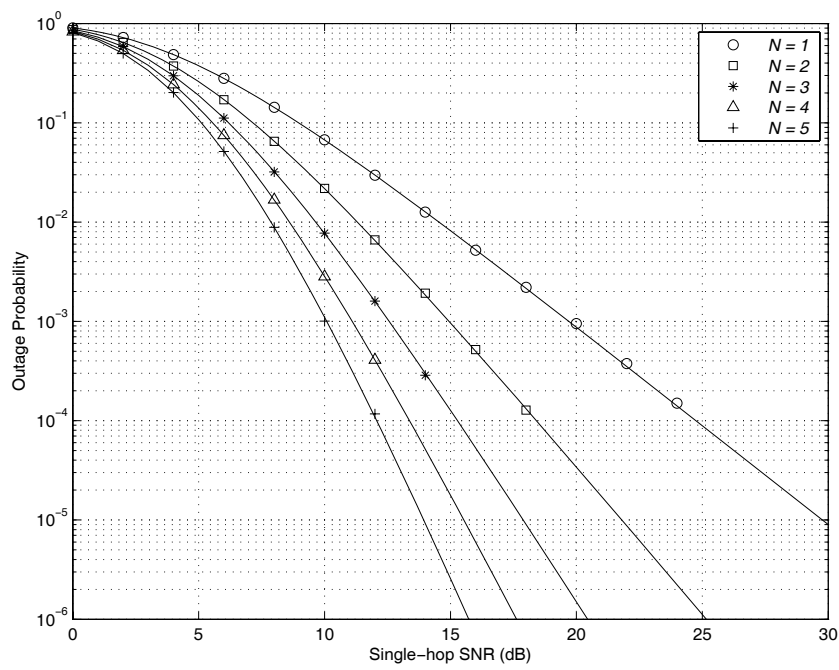

Fig. 3. Probability of outage of selection cooperative relaying (SCR) in Rayleigh fading.

channel gains). The solid curves represent the analytical results while the symbols represent the simulations; a close match is observed between the two. It is also observed that full diversity is achieved for the SCR scheme, i.e., the diversity order is equal to the number of relays plus one is obtained.

Fig. 4 shows the bit error rate of SR schemes for BPSK modulation. In this demonstration, a balanced relay network is adopted. The single-hop performance is also shown in this graph. In Fig 4, simulation results, indicated with symbols, match closely with the analytical ones shown as solid curves. In general, a diversity order equal to $N+1$ (for SCR) and $N$ (for SR) can be obtained for the $N$-relay network. This diversity order can be determined from the performance curves.

Figure 5 presents the ergodic capacity of the SCR and SR schemes. The capacity curves for SCR are shown with solid lines and for SR with dashed curves. The impact of using the direct path and cooperation is also obvious from this figure;

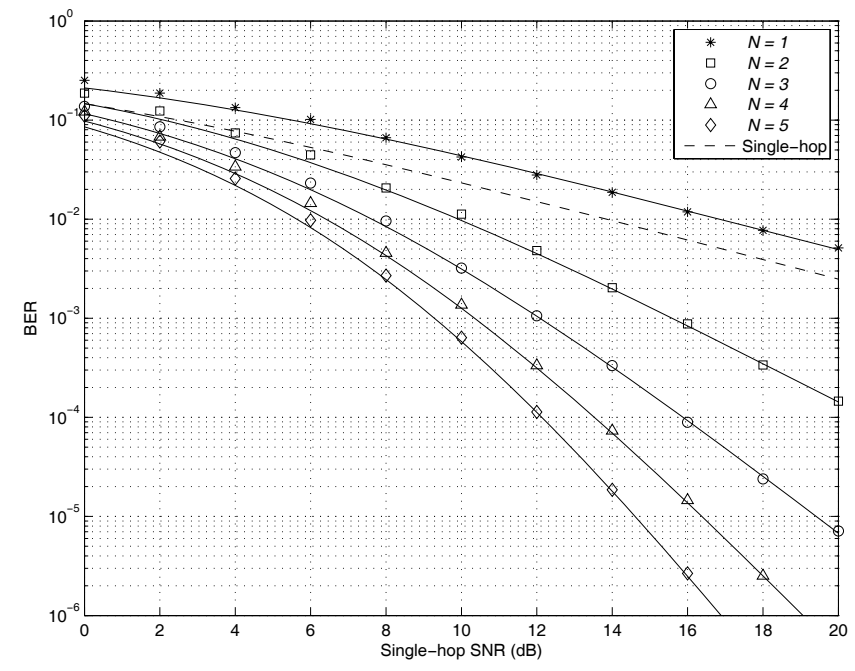

Fig. 4. BER performance of selection relaying (SR) scheme in Rayleigh fading, $\bar{\gamma}_{s d}=\bar{\gamma}_{s r}=\bar{\gamma}_{r d}(\mathrm{~dB})$.

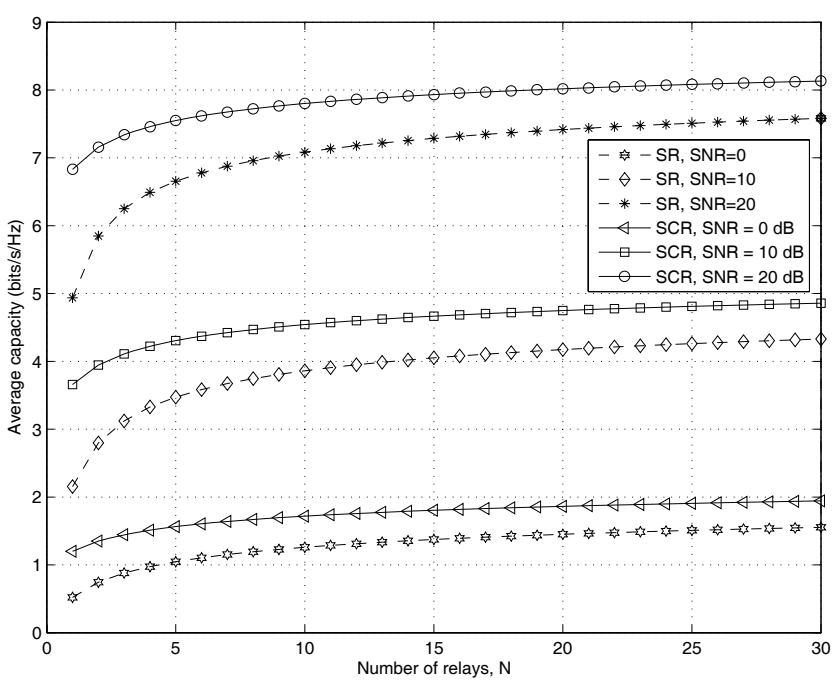

Fig. 5. Average capacity of selection relaying (SR) and selection cooperative relaying (SCR) in Rayleigh fading for different values of single-hop average SNR.

the capacity of the SCR is $11 \%$ greater than that of the SR scheme. A general observation is that the capacity saturates quickly with an increasing number of relays. This means that in reality not many relays are required to exploit the available system capacity.

The outage capacity behavior for the SR is shown in Fig. 6 for a fixed outage probability of $\epsilon=10^{-2}$ (solid curves) and $10^{-3}$ (dash curves) for a selected number of relays, $N=1,3$, and 5. Fig. 6 (a) shows the outage as a fraction of that of an AWGN channel. Presenting the figure this way helps to emphasis the diversity gain as well as to expose the impact of fading. Fig. 6 (b) on the other hand, shows the raw outage capacity and the effect of outage on capacity is clearly seen. The gain derived from the relays is also evident, by comparing the network of a single relay with that of two relays. From Fig. 6 (a), at $20 \mathrm{~dB}$, the capacity is increased from $6 \%$ of AWGN to $31 \%$ for an outage of $10^{-2}$, while an increase from $0.7 \%$ to $23.2 \%$ is recorded for an outage of $10^{-3}$. 

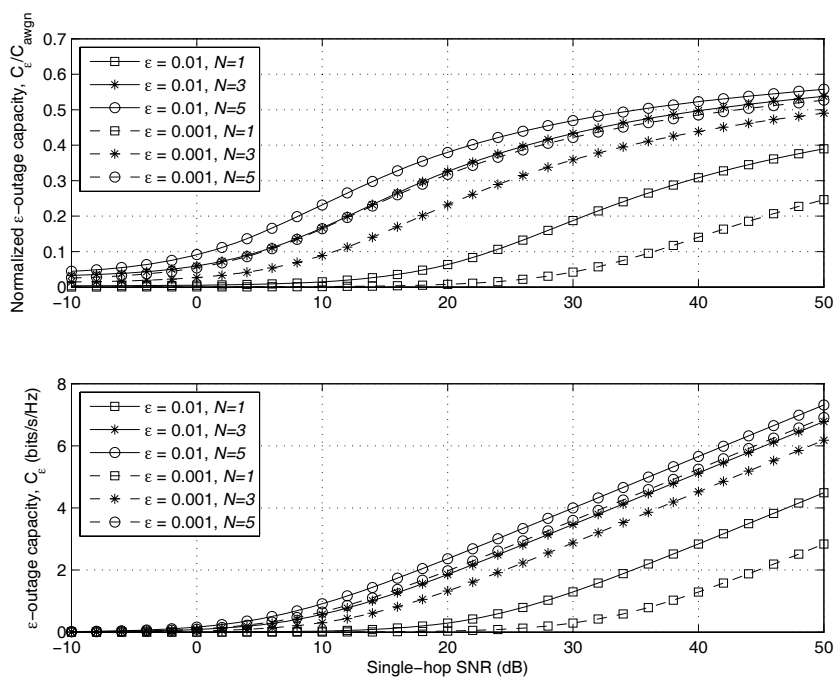

Fig. 6. The $\epsilon$-outage capacity performance of SR a) as a fraction of the AWGN capacity and b) the raw outage-capacity, $\bar{\gamma}_{s d}=\bar{\gamma}_{s r}=\bar{\gamma}_{r d}(\mathrm{~dB})$.

\section{Conclusion}

Most of the recent work on selection relaying has been focused on information theoretic analyses. High-SNR analyses are presented in most of these studies. In contrast, this paper has provided error rate analyses that are reasonably accurate over a large range of SNRs, most importantly in the low/medium SNR region. In addition, we have provided expressions for calculating the outage probability and ergodic capacity of the schemes. The outage capacity of the selection relaying technique has also been provided. It is worth noting that practical systems operate at considerably lower SNR values in comparison to the range where the high SNR analysis is accurate. The presented results may help network designers to make performance evaluations with a reasonable degree of accuracy in SNR regions of practical interest.

\section{APPENDIX I}

This appendix presents the derivation of the outage probability of the SCR scheme. The instantaneous rate can be expressed as $I=\frac{1}{2} \log _{2}(1+\beta)$ and therefore, the outage probability is

$$
\begin{aligned}
p_{\text {out }, \text { SCR }} & =\operatorname{Pr}(I<\mathcal{R})=\operatorname{Pr}\left(\log _{2}(1+\beta)<2 \mathcal{R}\right) \\
& =\int_{-\infty}^{a} \sum_{i=1}^{N}(-1)^{i-1}\left(\begin{array}{c}
N \\
i
\end{array}\right) \\
& \times \frac{2 i}{2 i \overline{\gamma_{0}}-\overline{\gamma_{1}}}\left(\exp \left[-\frac{\beta}{\bar{\gamma}_{0}}\right]-\exp \left[-\frac{2 i \beta}{\overline{\gamma_{1}}}\right]\right) d \beta,
\end{aligned}
$$

where $a=2^{2 \mathcal{R}}-1$. Interchanging the integral and summation operations, the integration can be performed as

$$
\begin{aligned}
& =\sum_{i=1}^{N}(-1)^{i-1}\left(\begin{array}{c}
N \\
i
\end{array}\right) \frac{2 i}{2 i \overline{\gamma_{0}}-\overline{\gamma_{1}}} \\
& \times \quad \int_{0}^{a}\left(\exp \left[-\frac{\beta}{\bar{\gamma}_{0}}\right]-\exp \left[-\frac{2 i \beta}{\overline{\gamma_{1}}}\right]\right) d \beta
\end{aligned}
$$

$$
\begin{aligned}
& =\sum_{i=1}^{N}(-1)^{i-1}\left(\begin{array}{c}
N \\
i
\end{array}\right) \frac{2 i}{2 i \overline{\gamma_{0}}-\overline{\gamma_{1}}}\left(\gamma_{0}-\frac{\overline{\gamma_{1}}}{2 i}\right. \\
& \left.+\frac{1}{2 i}\left[\overline{\gamma_{1}} \exp \left[-\frac{2 i a}{\overline{\gamma_{1}}}\right]-2 i \overline{\gamma_{0}} \exp \left[\frac{-a}{\overline{\gamma_{0}}}\right]\right]\right) .
\end{aligned}
$$

Finally,

$$
\begin{aligned}
p_{\text {out SCR }} & =\sum_{i=1}^{N}(-1)^{i-1}\left(\begin{array}{c}
N \\
i
\end{array}\right)\left(1+\frac{1}{2 i \overline{\gamma_{0}}-\overline{\gamma_{1}}}\right. \\
& \left.\times\left[\overline{\gamma_{1}} \exp \left(-\frac{2 i a}{\overline{\gamma_{1}}}\right)-2 i \overline{\gamma_{0}} \exp \left(\frac{-a}{\overline{\gamma_{0}}}\right)\right]\right),
\end{aligned}
$$

completing the derivation. Note that the factor $1 / 2$ is due to the half-duplex nature of the relaying schemes. For the SR, the outage probability is derived as follows:

$$
\begin{aligned}
p_{\text {out SR }}(\mathcal{R}) & =\operatorname{Pr}(I<\mathcal{R}) \\
& =\operatorname{Pr}\left(\frac{1}{2} \log _{2}\left(1+\min \left(\gamma_{s, r^{*}}, \gamma_{r^{*}, d}\right)\right)<\mathcal{R}\right) \\
& =\operatorname{Pr}\left(\min \left(\gamma_{s, r^{*}}, \gamma_{r^{*}, d}\right)<2^{2 \mathcal{R}}-1\right) \\
& =\operatorname{Pr}\left(\gamma_{1}<2^{2 \mathcal{R}}-1\right) ; \\
p_{\text {out }, S R} & =\int_{-\infty}^{2^{2 \mathcal{R}}-1} p\left(\gamma_{1}\right) d \gamma_{1} \\
& =\sum_{i=1}^{N}(-1)^{i-1}\left(\begin{array}{c}
N \\
i
\end{array}\right)\left(1-\exp \left[-\frac{2 i a}{\bar{\gamma}}\right]\right) .
\end{aligned}
$$

\section{APPENDIX II}

The capacity of SR can be obtained by taking $\bar{\gamma}_{0}$ to $-\infty$ (in $\mathrm{dB}$ ) in (14). Re-arranging the resulting expression produces (7).

\section{APPENDIX III}

After performing a number of manipulations, (5) can be expressed in the following form:

$$
P_{\text {out }, \mathrm{SR}}=\sum_{t=1}^{N}\left(\begin{array}{c}
N \\
i
\end{array}\right)(-p)^{t}+1,
$$

where $p=e^{-k}$ and $k=2\left(2^{2 \mathcal{R}}-1\right) / \bar{\gamma}$. For an outage $\epsilon$, the maximum supportable rate $C_{\epsilon}$ is obtained by solving the polynomial $\sum_{t=1}^{N}\left(\begin{array}{c}N \\ t\end{array}\right)(-p)^{t}+1-\epsilon=0$ to obtain

$$
C_{\epsilon}=\frac{1}{2} \log _{2}\left(1+\frac{\bar{\gamma}}{2} \log \left(\frac{1}{1-\epsilon^{\frac{1}{N}}}\right)\right) .
$$

\section{ACKNOWLEDGMENT}

The authors acknowledge valuable discussions with Dr. M. H. Ahmed of Memorial University, St. John's, Canada, and Dr. S. Al-Shebeili of King Saud University, Riyadh, Saudi Arabia. 


\section{REFERENCES}

[1] J. Laneman, D. Tse, and G. Wornell, "Cooperative diversity in wireless networks: efficient protocols and outage behavior," IEEE Trans. Inf. Theory, vol. 50, no. 11, pp. 3062-3080, Dec. 2004.

[2] S. Ikki and M. H. Ahmed, "Performance of multiple-relay cooperative diversity systems with best-relay selection over Rayleigh fading channels," EURASIP J. Wireless Commun. Networking, special issue wireless cooperative networks, vol. 2008, Article ID 580368, 7 pages, 2008.

[3] E. Beres and R. Adve, "Outage probability of selection cooperation in the low to medium SNR regime," IEEE Commun. Lett., vol. 11, no. 7, pp. 589-591, July 2007.

[4] E. Beres and R. Adve, "Selection cooperation in multi-source cooperative networks," IEEE Trans. Wireless Commun., vol. 7, no. 1, pp. 118-127, Jan. 2008.

[5] A. Bletsas, A. Khisti, D. Reed, and A. Lippman, "A simple cooperative diversity method based on network path selection," IEEE J. Sel. Areas Commun., vol. 24, no. 3, pp. 659-66, Mar. 2006.

[6] K. Woradit, T. Q. S. Quek, W. Suwansantisuk, H. Wymeersch, L. Wuttisittikulkij, and M. Z. Win, "Outage behavior of cooperative diversity with relay selection," in Proc. IEEE Global Telecommun. Conf. (GLOBECOM), New Orleans, LA, pp. 1-5, Nov. 2008.
[7] D. Tse and P. Viswanath, Fundamental of Wireless Communication. Cambridge, UK: Cambridge University Press, 2005.

[8] N. Balakrishnan and A. C. Cohen, Order Statistics and Inference: Estimation Methods. New York: Academic Press, 1991.

[9] L. Dai, B. Gui, and L. J. Cimini, "Selective relaying in OFDM multihop cooperative networks," in Proc. IEEE Wireless Commun. Networking Conf. (WCNC), Hong Kong, China, pp. 964-969, Mar. 2007.

[10] I. Gradshteyn and I. Ryzhik, Table of Integrals, Series, and Products. San Diego, CA: Academic Press, 1994.

[11] L. Long and E. Hossain, "Cross-layer optimization frameworks for multihop wireless networks using cooperative diversity," IEEE Trans. Wireless Commun., vol. 7, no. 7, pp. 2592-2602, July 2008.

[12] M. Hasna and M.-S. Alouini, "End-to-end performance of transmission systems with relay over Rayleigh-fading channels," IEEE Trans. Wireless Commun., vol. 2, no. 6, pp. 1126-1131, Nov. 2003.

[13] A. Adinoyi and H. Yanikomeroglu, "On the performance of cooperative wireless fixed relays in asymmetric channels," in Proc. IEEE Global Telecommun. Conf. (GLOBECOM), San Franscisco, CA, pp. 1-5, Nov. 2006. 